\title{
Auer-rod-like Inclusions in the Mature Neutrophils of a T-acute Lymphoblastic Leukemia Patient
}

Tomohiro Yabushita ${ }^{1}$, Kazuyuki Ueno ${ }^{2}$, Satoshi Yoshioka ${ }^{1}$ and Takayuki Ishikawa ${ }^{1}$

Key words: acute lymphoblastic leukemia, Auer-rod-like inclusions

(Intern Med 57: 3057-3058, 2018)

(DOI: 10.2169/internalmedicine.0756-18)

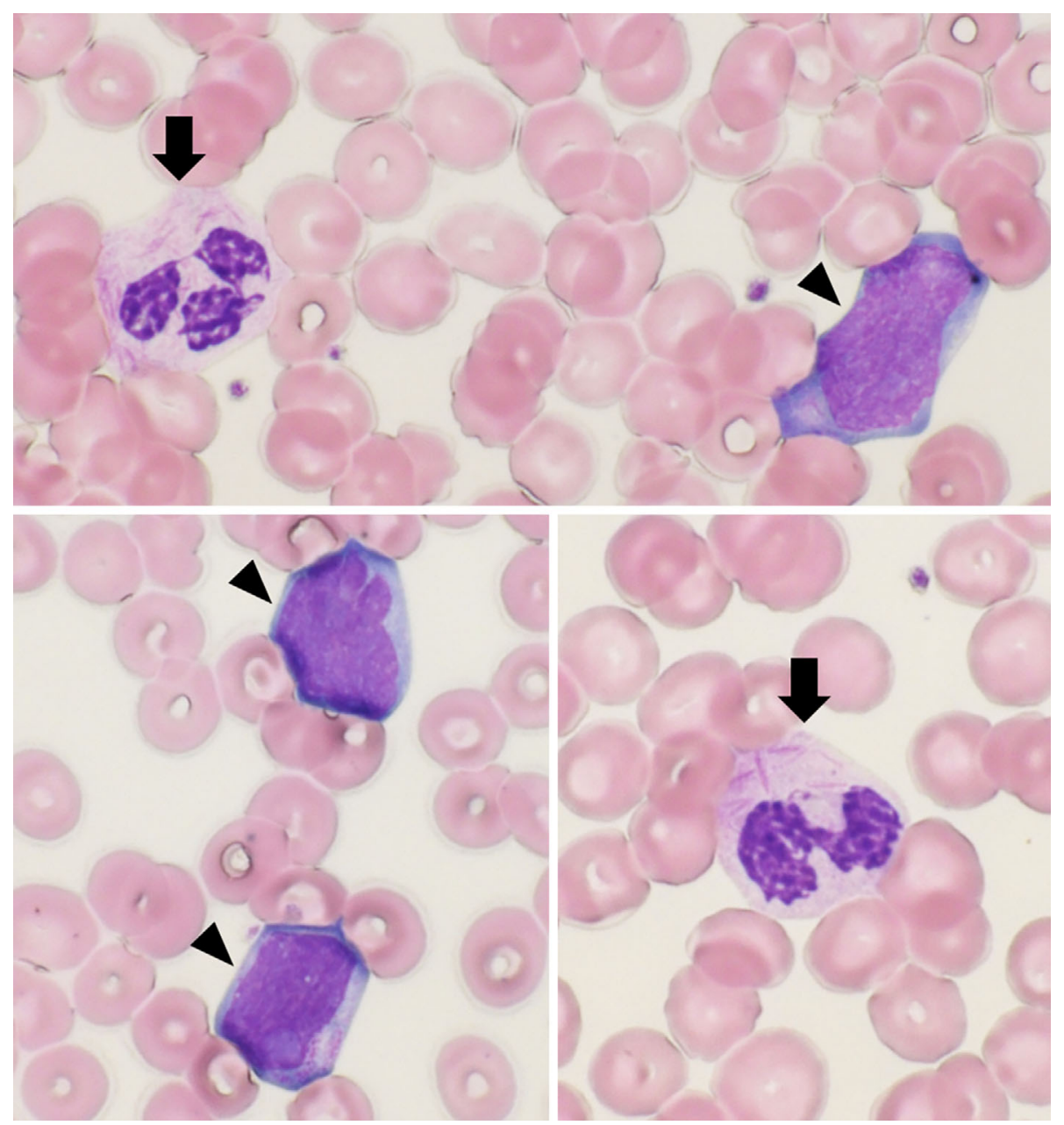

Picture.

A 70-year-old man presented with a 2-week history of fatigue. A hematological examination showed a leukocyte count of $16.2 \times 10^{9} / \mathrm{L}$, hemoglobin level of $104.0 \mathrm{~g} / \mathrm{L}$, and platelet count of $204.0 \times 10^{9} / \mathrm{L}$. A peripheral blood smear showed 53\% medium-sized blasts, with finely-dispersed chromatin and inconspicuous nucleoli (Picture, arrowhead).

${ }^{1}$ Department of Hematology, Kobe City Medical Center General Hospital, Japan and ${ }^{2}$ Department of Clinical Laboratory, Kobe City Medical Center General Hospital, Japan

Received: January 5, 2018; Accepted: March 4, 2018; Advance Publication by J-STAGE: June 6, 2018

Correspondence to Tomohiro Yabushita, t.yabushita527@kuhp.kyoto-u.ac.jp 
In addition, $7 \%$ of segmented neutrophils had cytoplasmic inclusions that closely resembled bundles of Auer rods (Picture, arrow). Bone marrow aspiration revealed that $90 \%$ of blasts were positive for CD2, cytoplasmic CD3, CD7, TdT, CD34, and CD99 and negative for CD19, CD20, and cytoplasmic myeloperoxidase on a flow cytometric analysis. A cytogenetic analysis showed a normal karyotype. These findings confirmed the diagnosis as T-cell acute lymphoblastic leukemia (ALL). Auer rods are morphological hallmarks that can be observed in acute myeloid leukemia or myelodysplastic syndrome. The possible mechanism underlying the Auer rod formation in this case is considered to be due to the concomitant abnormal hematopoiesis in the myeloid lineage. Auer-rod-like inclusions have rarely been observed in $\operatorname{ALL}(1,2)$. This case emphasizes that their presence does not exclude an ALL diagnosis.

The authors state that they have no Conflict of Interest (COI).

\section{References}

1. Dunphy CH, Chung D, Dunphy FR. Auer rod-like inclusions in adult common acute lymphoblastic leukemia. Human Pathol 25: 211-214, 1994

2. Dmitrienko S, Vercauteren S. Auer rods in mature granulocytes of a patient with mixed lineage leukemia. Blood 119: 4348, 2012.

The Internal Medicine is an Open Access journal distributed under the Creative Commons Attribution-NonCommercial-NoDerivatives 4.0 International License. To view the details of this license, please visit (https://creativecommons.org/licenses/ by-nc-nd/4.0/).

(C) 2018 The Japanese Society of Internal Medicine Intern Med 57: 3057-3058, 2018 